\title{
Focal laser ablation as clinical treatment of prostate cancer: report from a Delphi consensus project
}

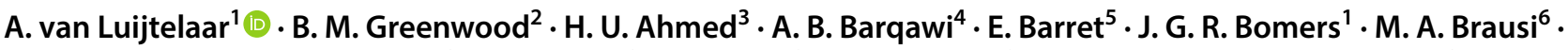 \\ P. L. Choyke ${ }^{7}$ - M. R. Cooperberg ${ }^{8} \cdot$ S. Eggener ${ }^{9}$ - J. F. Feller ${ }^{2} \cdot$ F. Frauscher ${ }^{10} \cdot$ A. K. George ${ }^{11} \cdot$ R. G. Hindley $^{12}$. \\ S. F. M. Jenniskens ${ }^{1} \cdot$ L. Klotz ${ }^{13} \cdot$ G. Kovacs ${ }^{14} \cdot$ U. Lindner ${ }^{15} \cdot$ S. Loeb ${ }^{16} \cdot$ D. J. Margolis ${ }^{17} \cdot$ L. S. Marks ${ }^{18} \cdot$ S. May ${ }^{2}$. \\ T. D. Mcclure ${ }^{19} \cdot$ R. Montironi ${ }^{20}$. S. G. Nour ${ }^{21} \cdot$ A. Oto ${ }^{22} \cdot$ T. J. Polascik ${ }^{23} \cdot$ A. R. Rastinehad ${ }^{24} \cdot$ T. M. De Reyke ${ }^{25}$. \\ J. S. Reijnen ${ }^{26}$. J. J. M. C. H. de la Rosette 27,28 . J. P. M. Sedelaar ${ }^{29}$ • D. S. Sperling ${ }^{30} \cdot$ E. M. Walser ${ }^{31}$ • J. F. Ward ${ }^{32}$. \\ A. Villers ${ }^{33} \cdot$ S. Ghai ${ }^{34} \cdot$ J. J. Fütterer ${ }^{1}$
}

Received: 25 September 2018 / Accepted: 10 January 2019 / Published online: 22 January 2019 (c) The Author(s) 2019

\begin{abstract}
Purpose To define the role of focal laser ablation (FLA) as clinical treatment of prostate cancer (PCa) using the Delphi consensus method.

Methods A panel of international experts in the field of focal therapy (FT) in PCa conducted a collaborative consensus project using the Delphi method. Experts were invited to online questionnaires focusing on patient selection and treatment of PCa with FLA during four subsequent rounds. After each round, outcomes were displayed, and questionnaires were modified based on the comments provided by panelists. Results were finalized and discussed during face-to-face meetings. Results Thirty-seven experts agreed to participate, and consensus was achieved on 39/43 topics. Clinically significant PCa (csPCa) was defined as any volume Grade Group 2 [Gleason score (GS) 3+4]. Focal therapy was specified as treatment of all csPCa and can be considered primary treatment as an alternative to radical treatment in carefully selected patients. In patients with intermediate-risk PCa (GS 3+4) as well as patients with MRI-visible and biopsy-confirmed local recurrence, FLA is optimal for targeted ablation of a specific magnetic resonance imaging (MRI)-visible focus. However, FLA should not be applied to candidates for active surveillance and close follow-up is required. Suitability for FLA is based on tumor volume, location to vital structures, GS, MRI-visibility, and biopsy confirmation.

Conclusion Focal laser ablation is a promising technique for treatment of clinically localized PCa and should ideally be performed within approved clinical trials. So far, only few studies have reported on FLA and further validation with longer follow-up is mandatory before widespread clinical implementation is justified.
\end{abstract}

Keywords Laser focal therapy $\cdot$ Focal laser ablation $\cdot$ Prostate cancer $\cdot$ Consensus $\cdot$ Delphi

\section{Introduction}

The incidence of prostate cancer $(\mathrm{PCa})$ is increasing with approximately 164,000 newly diagnosed cases in the US in 2018 [1]. Whole-gland therapy, i.e., radical prostatectomy (RP), external radiation therapy (RT), and brachytherapy are

Electronic supplementary material The online version of this article (https://doi.org/10.1007/s00345-019-02636-7) contains supplementary material, which is available to authorized users.

A. van Luijtelaar

Annemarijke.vanluijtelaar@ radboudumc.nl

Extended author information available on the last page of the article common treatment forms for PCa, providing excellent longterm efficacy but also come with treatment-related complications and side effects [2]. Studies reported urinary incontinence $(13.4 \%$ and $4.4 \%$, respectively), erectile function (EF) $(75.7 \%$ and $71.9 \%$, respectively) and bowel urgency (16.3\% and $31.3 \%$, respectively) within 5 years after RP or $\mathrm{RT}$, resulting in a measurable decrease in quality of life [3]. Minimally invasive techniques are used for organ-confined PCa and are a novel strategy for targeted treatment, while preserving healthy tissue and subsequently reduce treatmentrelated morbidity [4]. Currently, a wide variety of energy sources seem to be capable for FT, i.e., focal laser ablation (FLA) cryosurgery, high intensity-focused ultrasound 
(HIFU), radiofrequency, microwave, and irreversible electroporation/nanoknife (IRE) [5-7].

Accurate imaging of $\mathrm{PCa}$ in conjunction with the associated anatomy is crucial for an effective and successful treatment. Multiparametric magnetic resonance imaging (mpMRI) is preferred for cancer localization and treatment planning over other imaging modalities due to its excellent soft-tissue contrast and multi-planar, anatomical imaging. It is also suitable for image guidance during treatment and long-term follow-up after FT [8]. During image-guided treatments, MRI-based temperature-mapping provides real-time feedback of the thermal distribution in the prostate and thereby increasing both precision and control. Also, MRI-transrectal ultrasound (TRUS) fusion appears to be safe and feasible for FLA guidance and monitoring [9].

The clinical use of FLA for localized PCa is not established yet and further evaluation is needed before it can be recognized as FT. The FLA thermal ablation technique is applied with a laser fiber and by raising the temperature $>60{ }^{\circ} \mathrm{C}$, it results in direct focused cell death. Only few studies with small sample sizes and maximum follow-up of 1 year reported on the clinical use of FLA and, therefore, are lacking in long-term evidence [10-13]. However, they provide patient benefits supporting image-guided FLA as appealing technique and feasible and safe for minimalinvasive treatment, in men with low- and intermediate-risk $\mathrm{PCa}$ [Gleason score (GS) $\leq 3+4$ ] who are eligible for both active surveillance (AS) and radical treatment. Targeted biopsies from the ablation zone showed no recurrence within 3 months after FLA in $96 \%$. Systematic biopsies after 1 year showed a residual GS $\geq 3+3$ in $11 \%$. As a surrogate marker for efficacy, the mean prostate-specific antigen (PSA) has decreased by $40 \%$ following FLA $[14,15]$. The aim of this international collaborative consensus project was to define the role of image-guided FLA as potential clinical treatment in patients with clinically localized PCa using the Delphi consensus method among experts in the field [16].

\section{Method}

To achieve consensus among a panel of experts in the field, the Delphi method was used [16]. According to this method, online questionnaires were presented to participants during several rounds. The goal was to obtain consensus by reducing the range of answers after each round.

A systematic literature search was conducted using the PubMed database on "prostate" (and synonyms), "cancer" (and synonyms), "focal laser ablation" (and synonyms), "magnetic resonance imaging" (and synonyms), and "focal therapy" (and synonyms), with filters: Full text, to 2016/07/01, Humans, English. This search yielded 20 articles (Fig. 1), while only 10 articles were eligible for controversial topic selection.

Panelists were selected based on the literature search and peer recommendations. The survey was conducted and presented in four subsequent rounds between 28 November 2016 and 14 June 2017, using online questionnaire software (www.surveymonkey.com; San Mateo, USA). Questionnaires were modified after each round based on responses and feedback. A (statistical) summary of the previous rounds was provided allowing the experts to re-evaluate their opinion. The level of agreement to achieve consensus was set at $70 \%$ and descriptive statistics were used to determine the response rate of each topic. The selected topics from the literature search were demographics, patient characteristics, role of biopsy/imaging in FLA, tumor size, outcome, and genomics. Online Resource 1 displays an overview of the questionnaires.

During a face-to-face meeting at the American Urological Association Annual Meeting on 13 May 2017, the results of the first three rounds were presented and inconclusive topics were discussed. Remaining questions were reworded and presented in a fourth round. Final results of the project were discussed during a meeting at the Radiological Society of North America (RSNA) on 26 November 2017.

\section{Results}

Seventy-five international experts were invited, and 37 experts agreed to participate. Response rates were $100 \%$ (37/37), 70\% (26/37), 68\% (25/37), and 65\% (24/37) for rounds $1-4$, respectively. Fifty-one percent (19/37) were urologists, 38\% (14/37) (interventional) radiologists, 3\% (1/37) radiation oncologist, 3\% (1/37) researcher, 3\% (1/37) technical physician, and 3\% (1/37) engineer. Seventy-eight

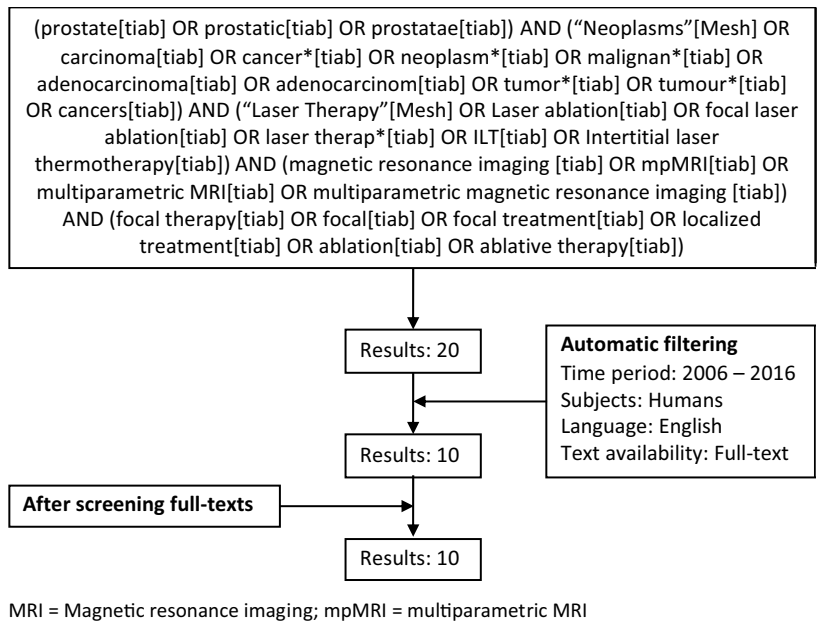

Fig. 1 Overview of systematic research 
percent (29/37) works in an academic hospital, 14\% (5/37) in private practice, and 8\% (3/37) in a major urban hospital. Eighty-six percent (32/37) reported $\mathrm{PCa}$ as their main field of expertise and 73\% (27/37) treat more than 150 patients per year. One participant (3\%) reported not to have treated any patients. Online Resource 2 contains the participants. Figure 2 shows the main results.

\section{Focal therapy}

Clinically significant PCa (csPCa) is defined as any volume GS $\geq 3+4(77 \%)$ and the lesion with both the largest volume and highest GS is considered the main index lesion (88\%). Focal therapy for de novo PCa is defined as treatment of csPCa while leaving insignificant lesions under AS (88\%). Subsequently, the same definition was used for FT as salvage treatment $(80 \%)$. It is recommended to use FT as primary treatment of $\mathrm{PCa}$ in well-selected patients as alternative to RP or RT (81\%). Focal laser ablation is optimal for targeting a specific focus $(95 \%)$, rather than quadrant ablation $(41 \%)$, hemi-gland ablation (24\%) or subtotal ablation of the prostate (14\%) (Fig. 3). Both, in-bore transperineal (100\%) or inbore transrectal (75\%) approach are recommended for FLA. There was no consensus on the MRI-TRUS fusion approach. Preservation of EF (97\%) and external urethral sphincter function (97\%) are important reasons for choosing FLA over radical treatment. However, FLA is not appropriate for any patient outside of clinical trials (73\%).

\section{Eligibility}

Eligibility for FLA is determined by GS (95\%), volume (81\%), location $(77 \%)$, and the number of MRI-visible and biopsy-confirmed cancers $(77 \%)$. Lower urinary tract

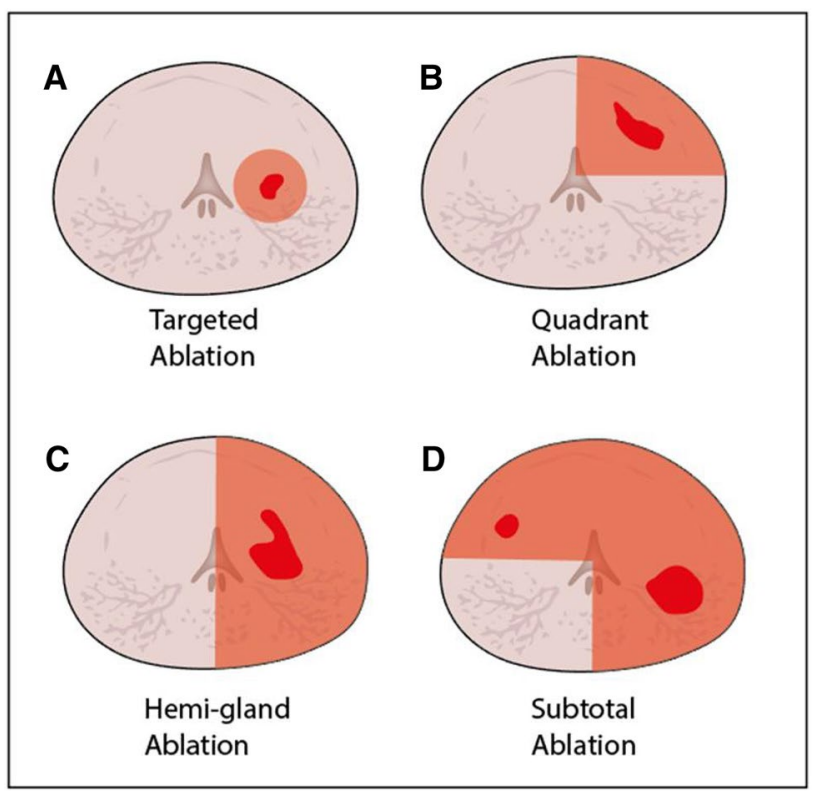

Fig. 3 Axial schematic views of prostate gland with cancer foci (in red) and ablation zones (in orange); a targeted ablation, b quadrant ablation, $\mathbf{c}$ hemi-gland ablation, and $\mathbf{d}$ subtotal ablation

symptoms are not a contraindication for FLA (89\%). The maximum prostate volume is not a primary determinant of eligibility for FLA (100\%). There was no consensus on patient selection and recommended PSA cut-off value reasonable for FLA. Subsequently, panelists were of the opinion that PSA levels should not be considered or used for inclusion (54\%). The maximum volume of cancer foci suitable for FLA should be based on MRI (81\%), however, it is recommended not to exceed a tumor volume of $10-15 \mathrm{~mL}$ (76\%). The panel recommended FLA in patients with low- and

Fig. 2 Summary of the results

\begin{tabular}{|c|c|}
\hline & 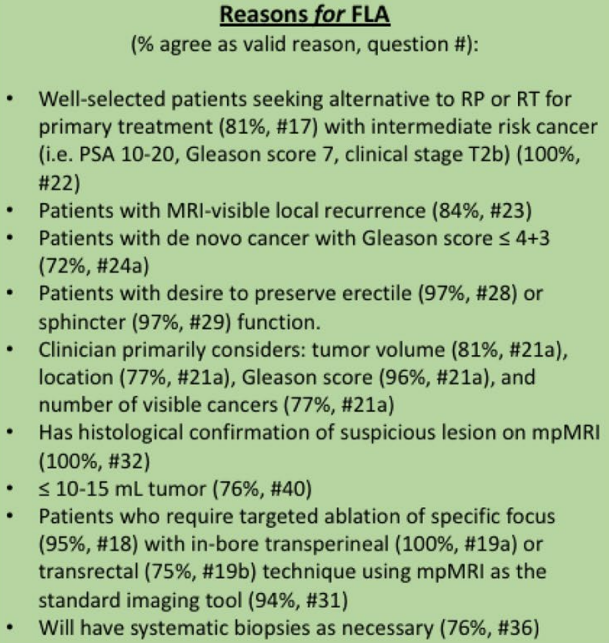 \\
\hline
\end{tabular}

\begin{tabular}{|l} 
Reasons for Not Having FLA \\
(\% agree as reason for no FLA, question \#): \\
- Patient is not involved in an official clinical trial \\
(73\%, \#26d) \\
Patients who are valid candidates for active \\
surveillance (73\%, \#26b) \\
Salvage patients without organ-confined disease \\
$(81 \%, \# 46)$
\end{tabular}

$\mathrm{RP}=$ Radical prostatectomy; $\mathrm{RT}=$ Radiotherapy; PSA = Prostate specific antigen; $\mathrm{MRI}$ = Magnetic resonance imaging; $\mathrm{mpMRI}=$ multiparametric $\mathrm{MRI}$ 
intermediate-risk $(100 \%)$ or in patients with MRI-visible local recurrence (84\%). It is also an acceptable strategy in patients with de novo PCa and GS $\leq 4+3$ (72\%). There was no consensus on the maximum GS in patients that require salvage therapy. However, salvage patients with only organconfined disease are suitable candidates for FLA (81\%). Patients who are AS candidate (GS 3+3) and otherwise do not require treatment are not eligible for FLA (73\%). Life expectancy is not a primary determinant of suitability for FLA (92\%). However, FLA should be offered when the life expectancy is less than 10 years, but only when treatment may delay local progression (96\%). It should be applied to patients whose life expectancy, excluding the PCa diagnosis, is greater than their expected disease-specific mortality (88\%).

\section{Biopsy and imaging}

Histological confirmation is still required prior to FLA in the presence of a suspicious lesion (PI-RADS 4 or 5) on mpMRI (100\%). Systematic biopsies remain necessary, even if an mpMRI suspicious lesion has already been sampled adequately by targeted biopsy (76\%). However, 12-core biopsy alone is insufficient for patient selection for FLA (73\%). The standard imaging tool for FT should be mpMRI (95\%) and MRI-TRUS software fusion biopsy (86\%) or inbore MRI-guided biopsy (86\%) are recommended to assess suspicious lesions. Cognitive-targeted biopsy was defined as not adequate to evaluate a lesion on mpMRI (88\%).

\section{Outcome}

According to the protocol, the prostate is regularly assessed by mpMRI and (systematic) biopsies after FLA. Treatment success is defined by a residual GS $3+3$ obtained by random biopsy of the treatment area in combination with a negative mpMRI (95\%). Residual in-field GS $\geq 3+4$ on TRUS-guided biopsy is defined as treatment failure (84\%), even with negative mpMRI (100\%). Subsequently, a GS $4+3$ based on infield TRUS-guided biopsy a negative mpMRI is also considered as treatment failure (97\%).

\section{Discussion}

Consensus projects are valuable tools in fields where clinical evidence is still developing. The provided statements and recommendations can contribute to standardization of a therapy for clinical utilization. The results of our consensus project reflect the opinion of 37 experts with experience in the field of FT for localized PCa. Image-guided FLA can be offered to carefully selected patients as alternative to radical treatment. It is most favorable for targeting a specific focus in patients with GS $\leq 4+3$ and MRI-visible, biopsy-proven PCa. Statements provided by this consensus project can be used as guidelines and recommendations for communitybased (interventional) radiologists and urologists performing or referring patients for FLA.

Several other consensus projects have been conducted to provide the opinion of experts in the field. Postema et al. defined FT as targeting all identified tumors and with the aim to eradicate all csPCa (GS $\geq 3+4)$ [17]. Donaldson et al. described FT as focal ablation of the main index or dominant lesion and recommended FT for both targeted and quadrant ablations. They did not agree on the maximum tumor volume suitable for FT [18]. Our panel assigned similar definitions to both treatments of de novo PCa and salvage therapy. Commonly, the largest lesion is simultaneously the highest GS and consequently the main index lesion [19]. Our experts considered the lesion with both the largest volume and highest GS as the main index lesion and agreed on targeted ablation of a specific focus rather than quadrant ablation with a maximum tumor volume of $10-15 \mathrm{~mL}$ on mpMRI. Considering the MRI-invisible portion of a tumor is likely to extend significantly as the tumor volume increases, ablation of a tumor of this size requires significant margins. There has been a shift in eligibility criteria in recent years from low- to intermediate-risk due to growing confidence in AS for low-risk cancers [20-22].

Currently, a wide variety of energy sources are available for FT. This project focused on one technique, being imageguided FLA. Transrectal FLA is performed under local anesthesia and can, therefore, be offered as outpatient procedure. It only takes a few minutes to create a sharp ablation zone which results in relatively short procedure times and quick patient recovery [11]. Furthermore, multiple ablations can be performed during one session and repeated treatments, i.e., FLA or secondary whole-gland therapy are still viable options after FLA. Number of studies concluded FLA as feasible and safe while preserving urinary and sexual function $[7,12,15]$. Despite the promising results, efficacy and oncological outcomes are not well established. Image-guided FLA is limited to centers with experienced (interventional) radiologists or urologists and the use is still sporadic due to high costs and lack of insurance coverage. Moreover, the FLA procedure is less optimal for large-sized PCa compared to other modalities. Appropriate patient selection and assessing the efficacy of MRI-guided interventions remain a controversy. Other studies agreed on oncologic efficacy similar to our panel and stated that insignificant cancers in post-treatment biopsies do not need further treatment [18, 24]. Our experts agreed on in-bore MRI-guided biopsy being adequate for assessing a suspicious lesion on mpMRI or post-treatment biopsies similar to other studies [25, 26]. Cognitive-targeted biopsy was defined as not adequate. Remarkably, Yaxley et al. showed no significant difference 
between in-bore MRI-guided biopsy or cognitive-targeted biopsy and described the importance of identifying the lesion on mpMRI [23].

Number of uncertainties were identified and remain topics for further research. The role of FLA as salvage therapy was discussed during a face-to-face meeting [27]. Valerio et al. reported five series $(n=115)$ with follow-up ranging between 3 and 90 months, receiving FT (i.e., cryosurgery, HIFU, and MRI-guided brachytherapy) after RT failure and described the pad-free continence (87-100\%), intact EF (29-40\%), and the absence of csPCa in 92\% [7]. Second, the use of both PSA and PSA-density (PSAD) as eligibility criteria for FLA remained undefined. Other studies considered a PSA $\leq 15 \mathrm{ng} / \mathrm{mL}$ eligible for FT and demonstrated PSAD as the most accurate predictor of PCa aggressiveness. The PSA was only used to predict seminal vesicle invasion $[28,29]$. Third, there was no consensus on the MRI-TRUS fusion approach for FLA as experience has been limited to date. Natarajan et al. allowed accurate ablation of the lesion with MRI-TRUS fusion $(n=11)$. However, in-field biopsy after 6 months showed a GS $3+3(n=3)$ or $\operatorname{csPCa}(n=4)$ [9].

Our study has several limitations. First, while using the Delphi method the results are based on the opinion of selected individuals and does not represent the PCa community at large. Expert selection can cause selection bias based on personal enthusiasm and preferences, since non-believers likely did not agree to participate. Also, all four questionnaires were not completed by every participating expert and the overall response rates were $65-100 \%$.

\section{Conclusion}

Focal laser ablation is a promising outpatient technique for treatment of clinically localized $\mathrm{PCa}$ in patients with GS $\leq 4+3$ and MRI-visible, biopsy-proven cancer. This study has shown that despite the large number of low- and intermediate-risk disease, patient selection and eligibility criteria for FLA need to be evaluated based on which patients will benefit the most before clinical implementation is justified.

Acknowledgements We would like to thank Dr. R. Toth for providing biostatistician input and graphics.

Author contributions AVL: protocol/project development, data collection or management, data analysis, and manuscript writing/editing. BMG: protocol/project development, data collection or management, and manuscript writing/editing. HUA: data collection or management. $\mathrm{AB}$ : data collection or management and manuscript writing/editing. EB: data collection or management. JGRB: data collection or management and manuscript writing/editing. MAB: data collection or management. PLC: data collection or management. MRC: data collection or management. SE: data collection or management. JFF: data collection or management. FF: data collection or management. AKG: data collection or management. RGH: data collection or management. SFMJ: data collection or management. LK: data collection or management. GK: data collection or management and manuscript writing/ editing. UL: data collection or management and manuscript writing/ editing. SL: data collection or management. DJM: data collection or management and manuscript writing/editing. LSM: data collection or management. STM: data collection or management. TDM: data collection or management. RM: data collection or management and manuscript writing/editing. SGN: data collection or management. AO: data collection or management. TJP: data collection or management and manuscript writing/editing. ARR: data collection or management. TMR: data collection or management and manuscript writing/editing. JSR: data collection or management and manuscript writing/editing. JMCHR: data collection or management and manuscript writing/editing. JPMS: data collection or management and manuscript writing/ editing. DSS: data collection or management. EMW: data collection or management. JFW: data collection or management. AV: data collection or management and manuscript writing/editing. SG: protocol/ project development, data collection or management, and manuscript writing/editing. JJF: protocol/project development, data collection or management, and manuscript writing/editing.

\section{Compliance with ethical standards}

Conflict of interest The following authors declare no interest of interest: A van Luijtelaar, BM Greenwood, AB Barqawi, E Barret, JGR Bomers, MA Brausi, PL Choyke, MR Cooperberg, JF Feller, F Frauscher, SFM Jenniskens, L Klotz, G Kovacs, U Lindner, LS Marks, S May, TD Mcclure, R Montironi, SG Nour, TJ Polascik, AR Rastinehad, TM De Reyke, JS Reijnen, JJMCH de la Rosette, JPM Sedelaar, DS Sperling, JF Ward, A Villers, JJ Fütterer. HU Ahmed received a research trial funding from Sophiris Bio., Sonacare inc., BTG/Galil and Trod Medical and accomplished paid proctoring and lecturing for Sonacare Inc. and BTG/Galil and is medical consultant for Sophiris Bio. S Eggener is advisor/consultant for Insightec and Sophiris and investigator, advisor and consultant for Profound Medical. AK George received a research trial funding from Nanospectra Biosciences. S Ghai received a research trial funding from CLS and is also PI of a MRgFUS study. RG Hindley received a research trial funding and accomplished paid proctoring and lecturing for Sonacare Inc. S Loeb is consulting with GE global research and Lilly. DJ Margolis is ad-hoc consultant for Blue Earth Diagnostics. A Oto received a research grant from Profound Healthcare and is on the Medical Advisory Board of Profound Healthcare. EM Walser is consulting with CLS in Sweden.

Ethical standard The manuscript does not involve human participants and/or animals. Informed consent was waived, and no medical ethical approval was needed for this study.

OpenAccess This article is distributed under the terms of the Creative Commons Attribution 4.0 International License (http://creativeco mmons.org/licenses/by/4.0/), which permits unrestricted use, distribution, and reproduction in any medium, provided you give appropriate credit to the original author(s) and the source, provide a link to the Creative Commons license, and indicate if changes were made.

\section{References}

1. Siegel RL, Miller KD, Jemal A (2018) Cancer statistics, 2018. CA Cancer J Clin 68(1):7-30 
2. Mottet N, Bellmunt J, Bolla $M$ et al (2017) EAU-ESTRO-SIOG guidelines on prostate cancer. Part 1: screening, diagnosis, and local treatment with curative intent. Eur Urol 71:618-629. https ://doi.org/10.1016/j.eururo.2016.08.003

3. Resnick MJ, Koyama T, Fan K-H et al (2013) Long-term functional outcomes after treatment for localized prostate cancer. $\mathrm{N}$ Engl J Med 368:436-445. https://doi.org/10.1056/NEJMoa1209 978

4. Eggener S, Salomon G, Scardino PT et al (2010) Focal Therapy for prostate cancer: possibilities and limitations. Eur Urol 58:5764. https://doi.org/10.1016/j.eururo.2010.03.034

5. Scheltema MJ, Chang JI, Böhm M et al (2018) Pair-matched patient-reported quality of life and early oncological control following focal irreversible electroporation versus robot-assisted radical prostatectomy. World J Urol 36:1383-1389. https://doi. org/10.1007/s00345-018-2281-z

6. Walker NAF, Shah MRC, Norris JM et al (2017) A comparison of time taken to return to baseline erectile function following focal and whole gland ablative therapies for localized prostate cancer: a systematic review. Urol Oncol Semin Orig Investig 66:1-10. https ://doi.org/10.1016/j.urolonc.2017.12.002

7. Valerio M, Ahmed HU, Emberton M et al (2014) The role of focal therapy in the management of localised prostate cancer: a systematic review. Eur Urol 66:732-751. https://doi.org/10.1016/j. eururo.2013.05.048

8. Bomers JGR, Sedelaar JPM, Barentsz JO, Fütterer JJ (2012) MRIguided interventions for the treatment of prostate cancer. Am J Roentgenol 199:714-720. https://doi.org/10.2214/AJR.12.8725

9. Natarajan S, Jones TA, Priester AM et al (2017) Focal laser ablation of prostate cancer: feasibility of magnetic resonance imagingultrasound fusion for guidance. J Urol 198:839-847. https://doi. org/10.1016/j.juro.2017.04.017

10. Lindner U, Weersink RA, Haider MA et al (2009) Image guided photothermal focal therapy for localized prostate cancer: phase I trial. J Urol 182:1371-1377. https://doi.org/10.1016/j. juro.2009.06.035

11. Lindner U, Lawrentschuk N, Weersink RA et al (2010) Focal laser ablation for prostate cancer followed by radical prostatectomy: validation of focal therapy and imaging accuracy. Eur Urol 57:1111-1114. https://doi.org/10.1016/j.eururo.2010.03.008

12. Oto A, Sethi I, Karczmar G et al (2013) MR imaging-guided focal laser ablation for prostate cancer: phase I trial. Radiology 267:932-940. https://doi.org/10.1148/radiol.12121652/-/DC1

13. Feller JF, Greenwood BM, Stafford RJ (2017) Transrectal laser focal therapy of prostate cancer. Imaging and focal therapy of early prostate cancer. Springer, Cham, pp 325-342

14. Lepor H, Llukani E, Sperling D, Fütterer JJ (2015) Complications, recovery, and early functional outcomes and oncologic control following in-bore focal laser ablation of prostate cancer. Eur Urol 68:924-926. https://doi.org/10.1016/j.eururo.2015.04.029

15. Eggener SE, Yousuf A, Watson S et al (2016) Phase II evaluation of magnetic resonance imaging guided focal laser ablation of prostate cancer. J Urol 196:1670-1675. https://doi.org/10.1016/j. juro.2016.07.074

16. Hsu CC, Sandford BA (2007) The Delphi technique: making sense of consensus. Pract Assess Res Eval 12(10):1-8. http://pareonline .net/getvn.asp? $\mathrm{v}=12 \& \mathrm{n}=10$
17. Postema AW, De Reijke TM, Ukimura O et al (2016) Standardization of definitions in focal therapy of prostate cancer: report from a Delphi consensus project. World J Urol 34:1373-1382. https:// doi.org/10.1007/s00345-016-1782-x

18. Donaldson IA, Alonzi R, Barratt D et al (2015) Focal therapy: patients, interventions, and outcomes-a report from a consensus meeting. Eur Urol 67:771-777. https://doi.org/10.1016/j.eurur o.2014.09.018

19. Weinreb JC, Barentsz JO, Choyke PL et al (2016) PI-RADS prostate imaging-reporting and data system: 2015, Version 2. Eur Urol 69:16-40. https://doi.org/10.1016/j.eururo.2015.08.052

20. Ahmed HU, Hindley RG, Dickinson L et al (2012) Focal therapy for localised unifocal and multifocal prostate cancer: a prospective development study. Lancet Oncol 13:622-632. https://doi. org/10.1016/S1470-2045(12)70121-3

21. Tay KJ, Cheng CWS, Lau WKO et al (2017) Focal therapy for prostate cancer with in-bore MR-guided focused ultrasound: twoyear follow-up of a phase I trial-complications and functional outcomes. Radiology 285:620-628. https://doi.org/10.1148/radio 1.2017161650

22. Klotz L, Vesprini D, Sethukavalan P et al (2015) Long-term follow-up of a large active surveillance cohort of patients with prostate cancer. J Clin Oncol 33:272-277. https://doi.org/10.1200/ JCO.2014.55.1192

23. Yaxley AJ, Yaxley JW, Pokorny MR et al (2017) Comparison between target magnetic resonance imaging (MRI) in-gantry and cognitively directed transperineal or transrectal-guided prostate biopsies for prostate imaging-reporting and data system (PI-RADS) 3-5 MRI lesions. BJU Int. https://doi.org/10.1111/ bju. 13971

24. de la Rosette J, Ahmed H, Barentsz J et al (2010) Focal therapy in prostate cancer-report from a consensus panel. J Endourol 24:775-780. https://doi.org/10.1089/end.2009.0596

25. Greenwood BM, Behluli MR, Feller JF, et al (2010) Transrectal interventional MRI: initial prostate biopsy experience. In: Proceedings SPIE 7625 medical imaging 2010: visualization, image-guided procedures, and modeling. https://doi. org/10.1117/12.847045

26. Felker ER, Lee-Felker SA, Feller J et al (2016) In-bore magnetic resonance-guided transrectal biopsy for the detection of clinically significant prostate cancer. Abdom Radiol 41:954-962. https://doi. org/10.1007/s00261-016-0750-7

27. Greenwood BM, May S, Feller JF (2016) Transrectal MR-guided laser focal therapy of recurrent prostate cancer in a patient postproton therapy: a case study. J Clin Oncol 34:e298-e298. https:// doi.org/10.1200/jco.2016.34.3_suppl.e298

28. Van Den Bos W, Muller BG, Ahmed H et al (2014) Focal therapy in prostate cancer: international multidisciplinary consensus on trial design. Eur Urol 65:1078-1083. https://doi.org/10.1016/j. eururo.2014.01.001

29. Sfoungaristos S, Perimenis P (2012) PSA density is superior than PSA and Gleason score for adverse pathologic features prediction in patients with clinically localized prostate cancer. Can Urol Assoc J 6:46-50. https://doi.org/10.5489/cuaj.11079 


\section{Affiliations}

A. van Luijtelaar ${ }^{1}$ (I) B. M. Greenwood ${ }^{2}$ - H. U. Ahmed ${ }^{3}$ - A. B. Barqawi ${ }^{4}$ - E. Barret ${ }^{5}$ - J. G. R. Bomers ${ }^{1}$ - M. A. Brausi ${ }^{6}$. P. L. Choyke $\cdot$ M. R. Cooperberg ${ }^{8} \cdot$ S. Eggener ${ }^{9}$. J. F. Feller ${ }^{2}$. F. Frauscher ${ }^{10} \cdot$ A. K. George ${ }^{11}$ - R. G. Hindley ${ }^{12}$.

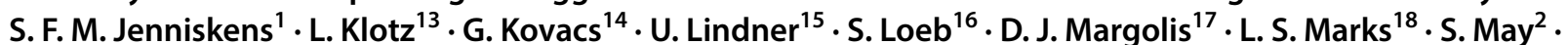
T. D. Mcclure ${ }^{19} \cdot$ R. Montironi ${ }^{20}$ - S. G. Nour ${ }^{21} \cdot$ A. Oto ${ }^{22}$-T. J. Polascik ${ }^{23} \cdot$ A. R. Rastinehad ${ }^{24} \cdot$ T. M. De Reyke ${ }^{25}$. J. S. Reijnen ${ }^{26}$. J. J. M. C. H. de la Rosette ${ }^{27,28}$. J. P. M. Sedelaar ${ }^{29}$. D. S. Sperling ${ }^{30}$ - E. M. Walser ${ }^{31}$. J. F. Ward ${ }^{32}$. A. Villers ${ }^{33} \cdot$ S. Ghai ${ }^{34} \cdot$ J. J. Fütterer ${ }^{1}$

1 Department of Radiology and Nuclear Medicine, Radboud University Medical Center, Nijmegen, The Netherlands

2 Desert Medical Imaging, Indian Wells, CA, USA

3 Department of Surgery, Imperial College London, London, UK

4 Division of Urology, Department of Surgery, University of Colorado Denver School of Medicine, Aurora, CO, USA

5 L'Institut Mutualiste Montsouris, Paris Descartes University, Paris, France

6 Department of Urology, AUSL Modena, Modena, Italy

7 Molecular Imaging Program, National Cancer Institute, Bethesda, MD, USA

8 University of California San Francisco, San Francisco, CA, USA

9 Department of Urology, University of Chicago Medical Center, Chicago, IL, USA

10 Medizinische Universität Innsbruck, Innsbruck, Austria

11 Urologic Oncology Branch, Center for Cancer Research, National Cancer Institute, National Institutes of Health, Bethesda, MD, USA

12 Department of Urology, Basingstoke Hospital, Hampshire Hospitals NHS Foundation Trust, Basingstoke, UK

13 Sunnybrook Health Sciences Centre, Toronto, ON, Canada

14 Interdisciplinary Brachytherapy Unit, University of Lübeck, Lübeck, Germany

15 Department of Urology, Kaplan Medical Center, Rehovot, Israel

16 Department of Urology and Population Health, New York University and Manhattan Veterans Affairs Medical Center, New York, NY, USA

17 Department of Radiology, Ronald Reagan-UCLA Medical Center, Los Angeles, CA, USA
18 Department of Urology, University of California-Los Angeles, Los Angeles, CA, USA

19 Department of Urology, New York Presbyterian-Weill Cornell Medical College, New York, NY, USA

20 Section of Pathological Anatomy, Polytechnic University of the Marche Region, School of Medicine, United Hospitals, Ancona, Italy

21 Department of Radiology and Imaging Sciences, Emory University School of Medicine, Atlanta, GA, USA

22 Department of Radiology, University of Chicago, Chicago, IL, USA

23 Department of Surgery, Duke University Medical Center, Durham, NC, USA

24 Department of Urology, Mount Sinai, New York, NY, USA

25 Department of Urology, Amsterdam UMC, Amsterdam, The Netherlands

26 Department of Radiology, Sørlandet Hospital, Kristiansand, Norway

27 Department of Urology, Istanbul Medipol University, Istanbul, Turkey

28 Amsterdam UMC University Hospital, Amsterdam, The Netherlands

29 Department of Urology, Radboud University Medical Center, Nijmegen, The Netherlands

30 Sperling Prostate Center, Manhattan, NY, USA

31 Department of Radiology, University of Texas Medical Branch, Galveston, TX, USA

32 Division of Surgery, Department of Urology, University of Texas, Houston, TX, USA

33 Department of Urology, Lille University Medical Center, Lille, France

34 University of Toronto, Toronto, ON, Canada 